\title{
Extreme gas kinematics in an off-nuclear HII region of SDSS J143245.98+404300.3
}

\author{
Bruno Rodríguez Del Pino ${ }^{1}$, Santiago Arribas ${ }^{1}$, Javier Piqueras López ${ }^{1}$, \\ Alejandro Crespo Gómez ${ }^{1}$, and José M. Vílchez ${ }^{2}$ \\ ${ }^{1}$ Centro de Astrobiología (CSIC-INTA), Torrejón de Ardoz, Madrid, Spain \\ e-mail: brodriguez@cab.inta-csic.es \\ ${ }^{2}$ Instituto de Astrofísica de Andalucía, (CSIC), Apdo. 3004, 18080 Granada, Spain \\ Received 20 June 2019 / Accepted 26 July 2019
}

\begin{abstract}
We present and discuss the properties of an ionized gas component with extreme kinematics in a recently reported off-nuclear HII region located at $\sim 0.8-1.0 \mathrm{kpc}$ from the nucleus of SDSS J143245.98+404300.3. The high-velocity-gas component is identified by the detection of very broad emission wings in the $\mathrm{H} \alpha$ line, with full width at half maximum $(F W H M) \geq 850-1000 \mathrm{~km} \mathrm{~s}^{-1}$. Such gas kinematics are outstandingly high compared to other HII regions in local galaxies and are similar to those reported in some star-forming clumps of galaxies at $z \sim 2$. The spatially resolved analysis indicates that the high-velocity gas extends at least $\sim 90 \mathrm{pc}$ and it could be compatible with an ionized outflow entraining gas at a rate between approximately seven and nine times faster than the rate at which gas is being converted into stars. We do not detect broad emission wings in other emission lines such as $\mathrm{H} \beta$, perhaps due to moderate dust extinction, nor in [NII] $\lambda \lambda 6548,6584$ or [SII] $\lambda \lambda 6717,6731$, which could be due to the presence of turbulent mixing layers originated by the impact of fast-flowing winds. The lack of spectral signatures associated to the presence of WolfRayet stars points towards stellar winds from a large number of massive stars and/or supernovae as the likely mechanisms driving the high-velocity gas.
\end{abstract}

Key words. galaxies: star formation - galaxies: kinematics and dynamics

\section{Introduction}

The presence of ionized gas moving at very high velocities in galaxies (full width at half maximum, $F W H M \geq 1000 \mathrm{~km} \mathrm{~s}^{-1}$ ) is generally associated to active galactic nuclei (AGN) and nuclear starbursts (e.g., Villar Martín et al. 2014; Heckman \& Borthakur 2016), although they have also been identified in HII galaxies (e.g., Terlevich et al. 2014, and references therein), especially in Green Peas (Amorín et al. 2012). However, gas moving at such high velocities has also been observed in off-nuclear HII regions, albeit in only a handful of cases reported so far in nearby galaxies (e.g., Diaz et al. 1987; Castañeda et al. 1990; Roy et al. 1991). Several mechanisms have been suggested to be responsible for the extreme kinematics observed in these off-nuclear regions, generally invoking the presence of a large number of massive stars and/or supernovae; however, there is still no consensus on their origin (Roy et al. 1992; Binette et al. 2009). Given the low number of detections (either by their low incidence or the observational limitations), the discovery of new ones and their detailed study is crucial to understanding the physical mechanisms associated to them. Moreover, the fact that ionized outflows appear to be common in off-nuclear star-forming regions of galaxies at $z \sim 2$ (Genzel et al. 2011) make their detection in nearby galaxies an important baseline for their study at high redshift.

The standard method to identify gas components with extreme kinematics in the spectra of galaxies is the detection of broad emission wings in lines such as $\mathrm{H} \alpha, \mathrm{H} \beta$, and [OIII] $\lambda 5007$, or those associated to Wolf-Rayet stars (Diaz et al.
1987; Izotov et al. 1996). However, observing them is challenging because they normally appear as low-luminosity pedestals superimposed on the emission from the host galaxy. In addition, since most spectroscopic observations generally target the central parts of galaxies, regions located away from them are systematically missed. In this sense, the advent of large integral field spectroscopic (IFS) surveys provide the appropriate spatial and spectral coverage to extend the search for these high-velocity gas components to a large number of galaxies.

In our recent work, Rodríguez del Pino et al. (2019; hereafter RDP19), where we explored the properties of ionized outflows in a parent sample of over 2700 galaxies from the Second Data Release (DR2; Abolfathi et al. 2018) of the Mapping Nearby Galaxies at Apache Point Observatory survey (MaNGA; Bundy et al. 2015), we discovered extreme highvelocity gas motions $\left(F W H M \geq 1000 \mathrm{~km} \mathrm{~s}^{-1}\right)$ in an off-nuclear HII region located at $\sim 1 \mathrm{kpc}$ from the center of the galaxy SDSS J143245.98+404300.3, at $z=0.0174$. The discovery was a result of the analysis of more than $5000 \mathrm{H} \alpha$-emitting regions in $\sim 1200$ systems, detecting signatures of ionized outflows in 105 individual regions. Here we analyze in more depth the MaNGA data and present new observations of this region performed with the Intermediate Dispersion Spectrograph and Imaging System (ISIS) on the $4.2 \mathrm{~m}$ William Herschel Telescope (WHT) of the Isaac Newton Group (ING) at the Roque de los Muchachos Observatory (La Palma, Spain). Throughout this work we adopt a $H_{0}=67.3 \mathrm{~km} \mathrm{~s}^{-1} \mathrm{Mpc}^{-1}, \Omega_{\mathrm{M}}=0.315$, $\Omega_{\Lambda}=0.685$ cosmology (Planck Collaboration XVI 2014). 


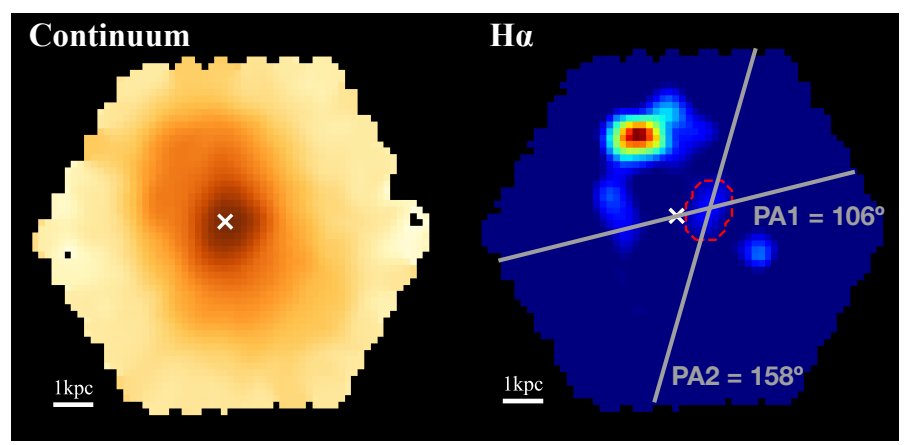

Fig. 1. Continuum (left) and $\mathrm{H} \alpha$ (right) emission maps of SDSS J143245.98+404300.3 obtained from the MaNGA data. The red dashed line highlights the position of the off-nuclear HII region where the high-velocity gas component has been found. The white cross indicates the photometric center of the galaxy. We have also highlighted in gray the two positions used for the long-slit spectroscopic observations with ISIS/WHT.

\section{Data and analysis}

In this work we use data from MaNGA DR2 for the galaxy SDSS J143245.98+404300.3, with a wavelength coverage from $3600 \AA$ to $10000 \AA$ and a resolving power ranging from $R \sim 1400$ to $R \sim 2600$ at short and long wavelengths, respectively. The spatial resolution element in the datacube is $2.5 \operatorname{arcsec}$ (FWHM), which corresponds to $0.875 \mathrm{kpc}$ at the redshift of the galaxy $(z=0.0174)$. In Fig. 1 we show the $\mathrm{H} \alpha$ emission and nearby continuum maps of the galaxy generated as in RDP19, highlighting (red, dashed line) the location of the off-nuclear HII region.

We also present and analyze follow-up long-slit spectroscopic observations taken with ISIS/WHT during the nights of March 9 and 10 of 2019. We used simultaneously the blue and red arms of ISIS with a 1.3 arcsec slit and the R600 grating, covering the wavelength ranges [4328-5584] A and [6392-7594] $\AA$. The spectral resolution (FWHM) measured on the sky lines are $0.95 \AA$ for the blue and $0.91 \AA$ for the red arm. The spectra were taken in several exposures of $40 \mathrm{~min}$ each in two position angles (Fig. 1). Reduction of the ISIS/WHT data was performed using standard IRAF tasks. For the analysis performed in this paper we use the five exposures ${ }^{1}$ where the broad component in the $\mathrm{H} \alpha$ line shows a more clear detection (three with PA1 and two with PA2). When these observations are combined, the total exposure time is $\sim 3.33 \mathrm{~h}$. We do not perform absolute flux calibration in the ISIS/WHT data because no standard star observation was included in the programme.

\subsection{Isolating the region hosting the broad emission}

Identification of the off-nuclear HII region in the MaNGA data was carried out in RDP19 by searching for individual $\mathrm{H} \alpha$ emitting regions in the host galaxy. The center of the region is located at $\sim 1 \mathrm{kpc}$ from the photometric center of the galaxy, measured by fitting a 2D Gaussian to the continuum emission in a region bluewards $\mathrm{H} \alpha$ (see Fig. 1). We extracted and combined the spectra from the $\mathrm{H} \alpha$-emitting region hosting the highvelocity gas using an aperture radius of $\sim 2 \operatorname{arcsec}(0.74 \mathrm{kpc})$, corresponding to an area of $\sim 13 \operatorname{arcsec}^{2}$. In Fig. 2 we show the

1 In the remaining exposures either the seeing conditions were not optimal or the slit was not placed correctly on the position of the clump.
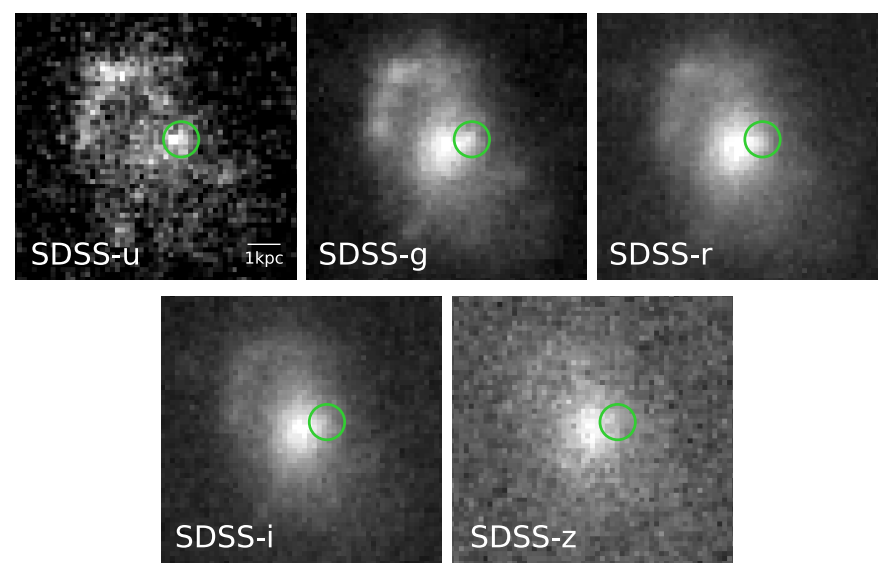

Fig. 2. SDSS ugriz images of SDSS J143245.98+404300.3 where we have highlighted the off-nuclear HII region hosting the ionized gas at high velocities.
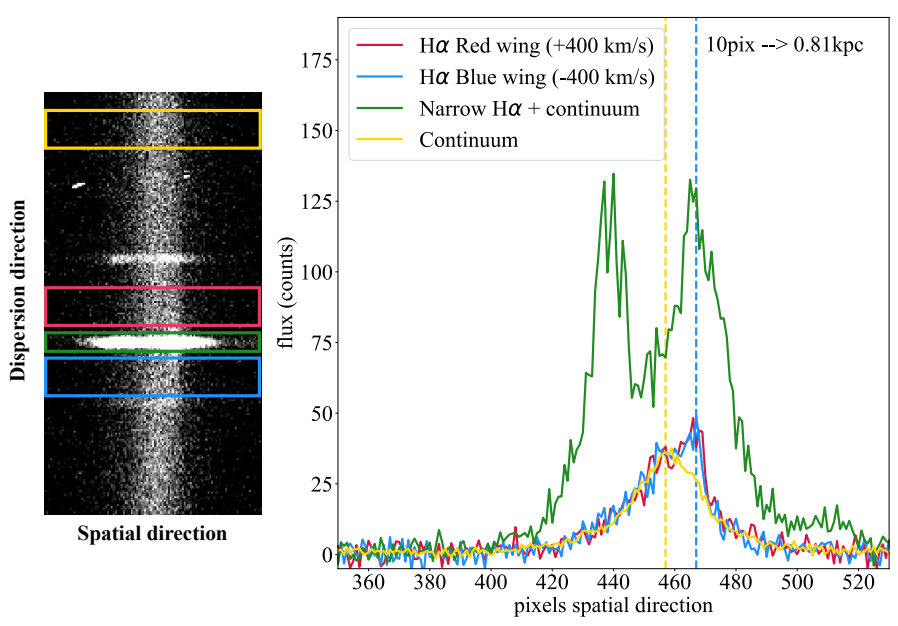

Fig. 3. Left: example of a $2 \mathrm{D}$ reduced image from the ISIS/WHT data obtained with the slit in the PA1 orientation. The colored regions are chosen to contain the light from the narrow component of $\mathrm{H} \alpha$ (green), the continuum emission (yellow), and the gas at velocities of $-400 \mathrm{~km} \mathrm{~s}^{-1}$ (blue) and $+400 \mathrm{~km} \mathrm{~s}^{-1}$ (red) with respect to the narrow component of $\mathrm{H} \alpha$ (systemic). Right: median light distribution for each of the regions selected in the left image. The vertical dashed lines mark the positions of the peaks of emission in the continuum and at $\pm 400 \mathrm{~km} \mathrm{~s}^{-1}$ with respect to the systemic emission.

SDSS ugriz images of SDSS J143245.98+404300.3, highlighting the location of the off-nuclear HII region identified in the MaNGA data. The images in the $u$ and $g$ bands (perhaps also in the $r$ band) show evidence for significant emission associated to the HII region.

To isolate the broad emission in the ISIS/WHT long-slit spectroscopic data we first extract the light profiles at different positions in the dispersion direction from the reduced 2D images, as shown in the left panel of Fig. 3. In this figure we represent the case of a single exposure taken with the slit in the PA1 orientation, where the center of the galaxy is also included (see Fig. 1). We have selected different regions in the dispersion direction, whose median light profiles are shown in the right panel. In this plot a clear peak of emission can be seen at high velocities (red and blue) that is shifted by approximately ten pixels $(\sim 2.2$ arcsec $)$ with respect to the peak of continuum emission (yellow), corresponding to the central parts of the galaxy. Such a spatial offset in pixels corresponds to $0.81 \mathrm{kpc}$, similar 

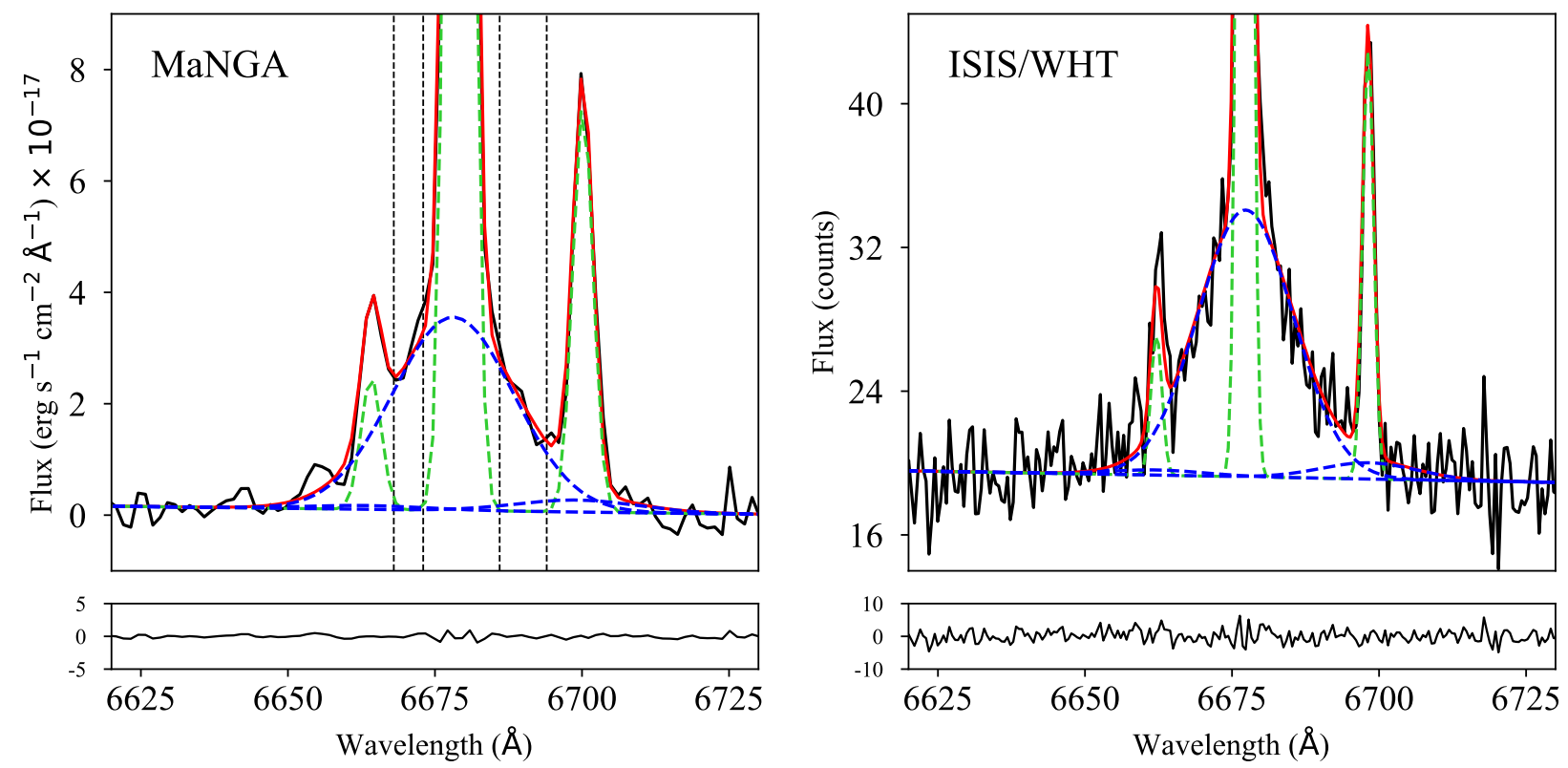

Fig. 4. Modeling of the MaNGA and ISIS/WHT spectra from the off-nuclear HII region identified in SDSS J143245.98+404300.3. Black solid lines represent the observed data. Green and blue dashed lines correspond to the narrow and broad components, respectively, and the red solid line is the sum of these two. The dashed vertical lines mark the wavelength ranges that are used to study the spatial extent of the broad emission in the MaNGA data (see Sect. 2.3).

to the distance measured in the MaNGA data $(\sim 1 \mathrm{kpc})$ and confirming the off-nuclear location of the broad emission. In this figure one can also see the emission from the other $\mathrm{H} \alpha$-emitting region on the opposite side of the galaxy that is also included in the slit. For the five exposures with better detections of the broad component, we extract and combine the spectra from the spatial positions (4-5 pixels) associated to the broad emission, which, taking into account the width of the slit and the spatial scale $\left(0.22 \operatorname{arcsec}\right.$ pixel $\left.^{-1}\right)$, correspond to extraction areas of $\sim 1-1.4 \operatorname{arcsec}^{2}$.

\subsection{Kinematics of the ionized gas}

We focus now on the study of the emission line profiles to constrain the kinematics of the ionized gas. For this analysis the spectra need to be corrected for stellar absorption, which can be specially severe in the $\mathrm{H} \beta$ line. For the MaNGA data, the modeling and subtraction of the stellar contribution was already done in RDP19. However, in the case of the nonflux-calibrated ISIS/WHT data, the continuum fitting is very uncertain due to the different flux levels of the blue and red arms of the ISIS instrument. Therefore, for the analysis of the blue part of the spectra, which includes the $\mathrm{H} \beta$ and [OIII] $\lambda 5007$ lines, we only use the MaNGA data, whereas for the red part, including $\mathrm{H} \alpha$, [NII], and [SII], we use both datasets. For the analysis of the $\mathrm{H} \alpha$ line we expect the effects from stellar absorption to be negligible.

We start by fitting the $\mathrm{H} \alpha$ and the neighboring [NII] $\lambda \lambda 6548,6584$ emission lines with a model consisting of two kinematic components: a narrow one to reproduce the systemic rotation of the host galaxy and a broad one to constrain the properties of the gas moving at high velocities. As in RDP19, we perform the modeling of the spectra using a Bayesian approach based on Markov chain Monte Carlo (MCMC) techniques, developed in the software EMCEE (Foreman-Mackey et al. 2013). The value adopted for each model parameter corresponds to the median of its probability density distribution and the errors are taken from the 16 th and 84 th percentiles.
In Fig. 4 we show the results from the spectral modeling ${ }^{2}$. In the MaNGA data, $F W H M_{\text {broad }}$ is $1074 \pm 160 \mathrm{~km} \mathrm{~s}^{-1}$, whereas in the ISIS/WHT data we measure $F W H M_{\text {broad }}=852 \pm 167 \mathrm{~km} \mathrm{~s}^{-1}$. In addition, we estimate the maximum velocity associated to the ionized gas, $V_{\max }=|\Delta V|+F W H M_{\text {broad }} / 2$, where $\Delta V$ is the velocity difference in $\mathrm{kms}^{-1}$ between the narrow and broad kinematic components. The values of $V_{\max }$ are $584 \pm 103 \mathrm{~km} \mathrm{~s}^{-1}$ and $432 \pm 198 \mathrm{~km} \mathrm{~s}^{-1}$ for the MaNGA and ISIS/WHT data, respectively. The values estimated for $F W H M_{\text {broad }}$ and for $V_{\max }$ in the two different datasets are fully consistent with each other, taking into account the errors, confirming the extraordinary nature of the kinematics in this region.

Based on the measured $\mathrm{H} \alpha$ fluxes on the MaNGA data and following the same method as in RDP19, the off-nuclear HII region has a total associated star formation rate (SFR) of $0.015 M_{\odot} \mathrm{yr}^{-1}$ (including narrow and broad components). The broad component comprises a significant fraction of the total flux in the $\mathrm{H} \alpha$ line, $~ 30 \%$ (MaNGA) and $48 \%$ (ISIS/WHT).

\subsection{Spatial extent and orientation of the broad emission}

To measure the spatial extent of the broad emission we take into account the fact that due to the velocities of the ionized gas, the light distribution observed at one wavelength (velocity) can be slightly shifted with respect to that observed at another (Carniani et al. 2015). Therefore, by estimating the centroid of the light distribution of the broad component at different wavelengths (velocities) we were able to estimate a minimum extent of the high-velocity gas. For this purpose, we use the MaNGA data to generate monochromatic images of the HII region at wavelengths where the broad component is dominant and that are located at both sides of the narrow $\mathrm{H} \alpha$ emission. In our case, these spectral windows are [6668-6673] $\AA$ and

2 We note that due to the larger extraction apertures employed (see Sect. 2.1), the $\mathrm{S} / \mathrm{N}$ in the MaNGA data is higher and the narrow lines are broader (a larger area encompasses higher dispersion velocities). 


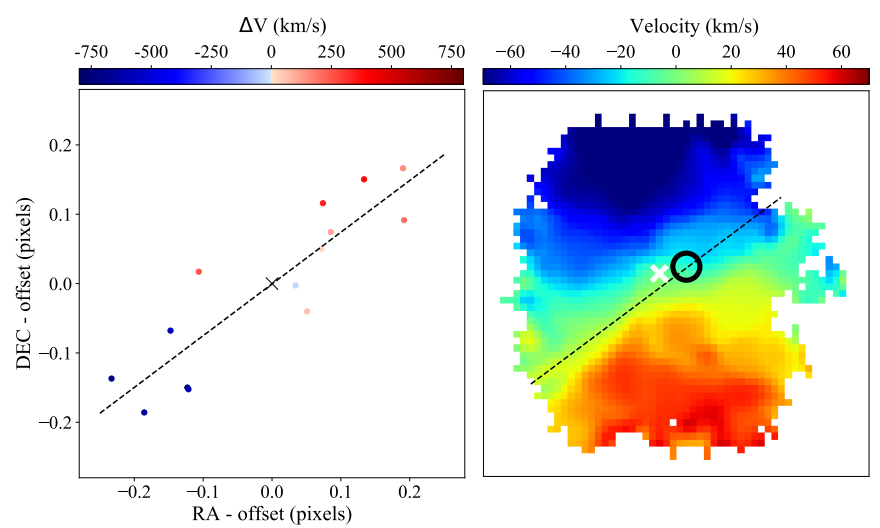

Fig. 5. Left: positions of the centroid of emission at different velocities (wavelengths) around the HII region in the MaNGA data. The different spatial location of the receding (red) and preceding (blue) light indicate that the emission is spatially resolved, corresponding to a size of $\sim 90 \mathrm{pc}$. The black cross indicates the centroid of emission at the wavelength of reference, $6685 \AA(\Delta V=0)$. Right: velocity field of galaxy SDSS J143245.98+404300.3. The black circle marks the location of the HII region. The dashed line corresponds to the orientation of the high-velocity gas, as determined in the left panel. As in Fig. 1 the white cross marks the photometric center of the galaxy.

[6686-6694] $\AA$ (dashed, vertical lines in Fig. 4), and the wavelength of reference is set at $6685 \AA$. In this way, we cover a spectral range $\Delta \lambda=26 \AA$, which corresponds to $\sim 1200 \mathrm{~km} \mathrm{~s}^{-1}$. We then fit each monochromatic image with a $2 \mathrm{D}$ Gaussian function to find the centroid of the light distribution at each wavelength (velocity). The result of this analysis is shown in Fig. 5 (left panel), where we can see that the receding light (red) is spatially shifted with respect to the light that is preceding (blue). Using the maximum distance between the points we estimate a minimum projected extent of the high-velocity gas of $\sim 90 \mathrm{pc}$ $(\sim 0.28 \mathrm{arcsec})^{3}$. In the right panel of Fig. 5 we show the velocity map of the host galaxy and, on top of it, the direction of the high-velocity gas as obtained in the left panel of the same figure. The orientation of the high-velocity gas is slightly different to that of the projected kinematic semi-minor axis, although the kinematics in the central regions of the galaxy are quite irregular.

\subsection{Search for broad emission in other spectral lines}

We now explore the presence of broad emission wings in other spectral lines available in our spectra. In the case of [NII] $\lambda 6584$, as shown in Fig. 4, a secondary kinematic component is very weak, although its detection is complicated due to the wide wings of $\mathrm{H} \alpha$. Based on the results from the MCMC analysis, the probability of the broad component in [NII] $\lambda 6584$ having $S / N>5$ is $\sim 24 \%$ in MaNGA and $\sim 64 \%$ in ISIS/WHT, and the estimated $[\mathrm{NII}] / \mathrm{H} \alpha$ ratio for the broad component in both data sets is $\sim 0.06$. The detection of broad components in [SII] $\lambda \lambda 6717,6731$ is less likely $(\operatorname{prob}(S / N>5)<1 \%)$.

\footnotetext{
3 We note that differential atmospheric refraction could also produce a wavelength shift in the position of the centroid. For the airmass of the MaNGA observations, 1.06, taken from the MaNGA DR2 cata$\log$ (Abolfathi et al. 2018), the corresponding differential atmospheric refraction between $6500 \AA$ and $7000 \AA$ is $0.03 \operatorname{arcsec}$ (Filippenko 1982). Given that we are using a much smaller wavelength window, $\Delta \lambda=26 \AA$, differential atmospheric refraction cannot produce the spatial variations of $\sim 0.28$ arcsec that we observe.
}

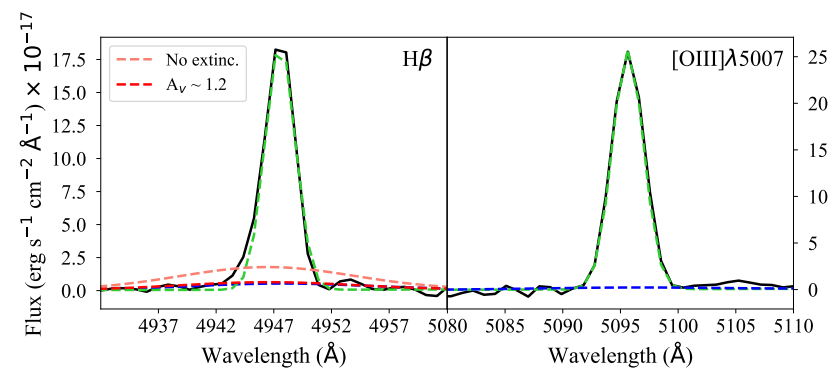

Fig. 6. MaNGA spectrum of the HII region around the $\mathrm{H} \beta$ (left) and [OIII] (right) lines, with the result from the spectral fit (blue and green dashed lines) fixing the kinematics to those found in $\mathrm{H} \alpha$. In the left panel we also show the expected $\mathrm{H} \beta$ profile for a case with no extinction (light red) and one with $A_{V} \sim 1.2$ (dark red), indicating that a moderate extinction could prevent the detection of the broad component in $\mathrm{H} \beta$.

For the analysis of the $\mathrm{H} \beta$ and [OIII] $\lambda 5007$ lines we use the MaNGA spectra, once corrected for stellar absorption. To explore the presence of broad emission wings we fit these lines with a model with the same kinematics obtained in the fit to the $\mathrm{H} \alpha$ and [NII] lines, allowing the fluxes of the two kinematic components to vary. The spectral modeling of $\mathrm{H} \beta$ and [OIII] $\lambda 5007$ is shown in Fig. 6. The probabilities of detecting a broad component in $\mathrm{H} \beta$ and [OIII] $\lambda 5007$ with $S / N>5$ are $39 \%$ and $11 \%$, respectively, whereas the ratio [OIII] $\lambda 5007 / \mathrm{H} \beta$ is $\sim 0.97$. In Fig. 6 we also evaluate the effects of extinction and show that a moderate extinction, $A_{V} \sim 1.2$ or larger, would be enough to erase the signature of a broad component in $\mathrm{H} \beta$. In RDP19 we estimated an extinction for the narrow component of $A_{V} \sim 0.3$, although the median extinction values for narrow and broad components in star-forming regions are much higher, $A_{V} \sim 1.7$.

In some works, the origin of broad $\mathrm{H} \alpha$ wings in HII regions has been related to the detection of a large number of WolfRayet stars (Sargent \& Filippenko 1991; Izotov et al. 1996), or to contamination from broad stellar components originating in supergiants or luminous blue variables (e.g., Terlevich et al. 1996). However, as shown in Fig. 7, the MaNGA, continuum subtracted spectrum from our off-nuclear HII region does not show signatures of broad emission in spectral lines associated to Wolf-Rayet stars (HeII $\lambda 4686$, NII $\lambda 4640$ and CII $\lambda 4650$ ) or to the presence of supergiants or luminous blue variables (HeI lines). Following the method explained in Guseva et al. (2000), given the $\mathrm{S} / \mathrm{N}$ in the spectral region around HeII $\lambda 4686(\sim 30)$ and the luminosity distance to the galaxy $(\sim 80 \mathrm{Mpc})$, we could only detect populations of more than $\sim 200$ Wolf-Rayet stars, providing an upper limit on the number of this type of stars expected to be associated to our HII region.

\section{Discussion and conclusions}

The extremely high velocities ( $F W H M \geq 850-1000 \mathrm{~km} \mathrm{~s}^{-1}$ ) of the ionized gas in the off-nuclear HII region of SDSS J143245.98+404300.3 indicate that we are witnessing an exceptional event, especially given the low SFR $\left(0.015 M_{\odot} \mathrm{yr}^{-1}\right)$ associated to it. In fact, such velocities are more than a factor of three larger than those observed in star-forming clumps with similar SFRs in local ULIRG/s (Arribas et al. 2014) and similar to those found in galaxies at $\sim 2$ (Genzel et al. 2011), where the SFRs are much higher $\left(3-66 M_{\odot} \mathrm{yr}^{-1}\right)$. Any connection with nuclear activity is discarded given the distance $(\geq 0.8-1 \mathrm{kpc})$ that separates the region from the center of the host galaxy. The extended nature of the region hosting gas at high velocities $(\geq 90 \mathrm{pc})$, 


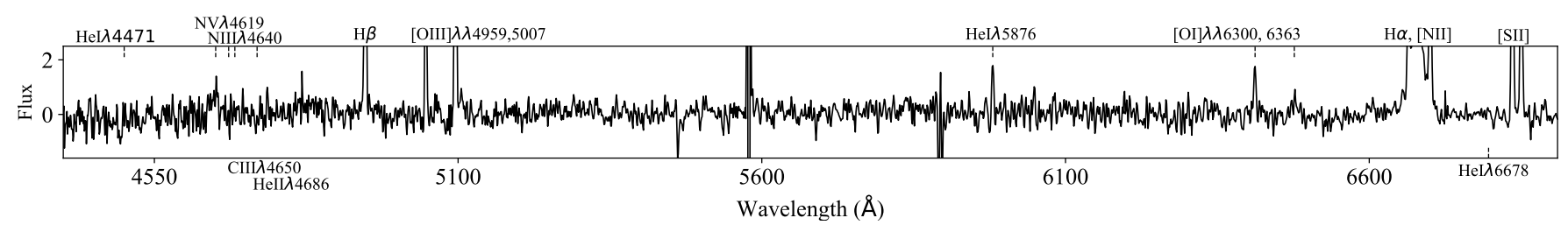

Fig. 7. Full observed spectrum of the off-nuclear HII region. We mark the wavelengths of the spectral lines associated to Wolf-Rayet stars (around $4700 \AA ̊$ Abserved wavelength) and the HeI lines, which could be indicative of broad stellar components. The lack of broad features in these spectral lines indicates that the origin of the $\mathrm{H} \alpha$ broad wings is not related to Wolf-Rayet stars or due to contamination from supergiants or luminous blue variables.

similar to that found in other HII regions (Castañeda et al. 1990; González Delgado \& Pérez 2000), implies that it cannot originate from a single source (e.g., supernova or AGN) and a large number of stellar objects are probably contributing. The detection of significant emission in the off-nuclear HII region in the $u$ and $g$ SDSS bands (see Fig. 2) is also consistent with the presence of a large young stellar population originating from a recent burst of star formation. The host galaxy does not show any indication of a previous interaction with nearby objects and its morphology was considered to be "uncertain" in the visual classification of Galaxy Zoo 1 (Lintott et al. 2008, 2011). Based on the images, the galaxy is quite symmetrical, with some signs of spiral arms hosting star formation.

The fact that the high-velocity gas moves in a well-defined orientation at positive (redshifted) and negative (blueshifted) velocities with respect to the systemic emission could be interpreted as outflowing gas that moves preferentially perpendicular to the galactic disk due to the presence of surrounding material, only allowing the gas to move in directions with less obstacles. In such a case, we could evaluate the local impact of the outflow by comparing the rate at which gas is being entrained by the outflow, that is, the outflowing mass rate, $\dot{M}$, with the rate at which gas is being converted into stars, namely the SFR in the region. The ratio between these two parameters is the so-called "massloading factor", $\eta=\dot{M}$ /SFR. To estimate approximated values of $\eta$ we follow the procedure explained in Arribas et al. (2014, Eqs. (2)-(6)), assuming that the gas moves at maximum velocity and an electron density of the outflowing gas of $\sim 300 \mathrm{~cm}^{-3}$ (median value in star-forming galaxies found in RDP19). We derive $\eta$ values of $\sim 9$ and $\sim 7$ for the MaNGA and ISIS/WHT data, respectively, indicating that the outflow is probably producing significant quenching of star formation. The $\eta$ values derived here are extremely high, larger than those found in local starforming galaxies (RDP19), local ULIRGs (Arribas et al. 2014), dwarf and starburst galaxies (Martin 1999), and $z \sim 2$ starforming galaxies (Genzel et al. 2011), which demonstrates the outstanding nature of the high-velocity gas studied here.

Despite the detection of a secondary, broad kinematic component in the $\mathrm{H} \alpha$ line, no other emission lines in our spectra show clear signs of broad emission. Although the probability of detecting a broad component in $\mathrm{H} \beta$, [OIII] $\lambda 5007$, and [NII] $\lambda 6584$ is low (see Sect. 2.4), their associated fluxes would be consistent with ionization coming from star formation, albeit with very low metallicities, from their position in the traditional BPT diagram (Baldwin et al. 1981). A lack of broad components in [NII] $\lambda \lambda 6548,6584$ and [SII] $\lambda \lambda 6717,6731$ was also observed in NGC 2363, which was suggested to be produced by a high ionization parameter (leading to gas dominated by high excitation species) associated to the presence of turbulent mixing layers caused by the impact of fast-flowing winds (Binette et al.
2009). However, there is still no consensus on what could be the origin of such strong winds. In many cases, the origin of these high velocities has been associated to the presence of a large number of Wolf-Rayet stars (Sargent \& Filippenko 1991; Izotov et al. 1996; González Delgado \& Pérez 2000), since these stars can experience strong mass losses and large terminal velocities (e.g., Dodorico \& Rosa 1981). However, in other cases where no Wolf-Rayet signatures are detected, a large number of massive $\mathrm{O}$, B stars and/or supernovae are invoked to explain the large velocities observed (Castañeda et al. 1990; Binette et al. 2009). In our case, the lack of broad emission around the HeII $\lambda 4686$, NII $\lambda 4640$, and CII $\lambda 4650$ lines points towards a lack of a significant number of Wolf-Rayet stars, therefore indicating that the origin of the gas at very high velocities is probably related to stellar winds from a large number of massive $\mathrm{O}, \mathrm{B}$ stars and/or supernovae originating from a recent burst of star formation.

In summary, here we present a detailed analysis of the properties of the high-velocity gas $\left(F W H M \geq 850-1000 \mathrm{~km} \mathrm{~s}^{-1}\right)$ in an off-nuclear HII region of the galaxy SDSS J143245.98+404300.3 reported in RDP19. First discovered using MaNGA data, we have further confirmed the presence of the high-velocity gas using data from ISIS/WHT. The gas kinematics are much higher than those observed in local HII regions and similar to those in galaxies at $z \sim 2$. Our analysis also indicates that the high-velocity gas is probably associated to an ionized outflow entraining gas at a rate between approximately seven and nine times faster than the rate at which gas is being converted into stars in that region. Both the kinematics of the ionized gas and its off-nuclear origin make this a very singular event, with only a few similar cases previously reported in the literature. The lack of connection to nuclear starburst or AGN activity and to Wolf-Rayet stars suggests that the origin of the large kinematics observed is connected to the presence of a large number of massive stars and/or supernovae, although the actual origin is still unknown.

Acknowledgements. We acknowledge the anonymous referee for constructive comments and suggestions. BRP, SA and AC acknowledge support from the Spanish Ministerio de Economía y Competitividad through the grant ESP201568964-P and ESP2017-83197. JPL acknowledges support from the Spanish Ministry of Economy and Competitiveness through grant AYA2017-85170-R. This work is based on observations taken with ISIS/WHT during the nights of March 9th and 10th of 2019 in visitor mode as part of the observing program 064-WHT6/19A. Funding for the Sloan Digital Sky Survey IV has been provided by the Alfred P. Sloan Foundation, the U.S. Department of Energy Office of Science, and the Participating Institutions. SDSS-IV acknowledges support and resources from the Center for High-Performance Computing at the University of Utah. The SDSS web site is www.sdss.org. This research made use of Astropy, a community-developed core Python package for Astronomy (Astropy Collaboration 2018). This research has made use of the NASA/IPAC Extragalactic Database (NED) which is operated by the Jet Propulsion Laboratory, California Institute of Technology, under contract with the National Aeronautics and Space Administration. 


\section{References}

Abolfathi, B., Aguado, D. S., Aguilar, G., et al. 2018, ApJS, 235, 42 Amorín, R., Vílchez, J. M., Hägele, G. F., et al. 2012, ApJ, 754, L22 Arribas, S., Colina, L., Bellocchi, E., Maiolino, R., \& Villar-Martín, M. 2014, A\&A, 568, A14

Astropy Collaboration (Price-Whelan, A. M., et al.) 2018, AJ, 156, 123

Baldwin, J. A., Phillips, M. M., \& Terlevich, R. 1981, PASP, 93, 5

Binette, L., Drissen, L., Ubeda, L., et al. 2009, A\&A, 500, 817

Bundy, K., Bershady, M. A., Law, D. R., et al. 2015, ApJ, 798, 7

Carniani, S., Marconi, A., Maiolino, R., et al. 2015, A\&A, 580, A102

Castañeda, H. O., Vilchez, J. M., \& Copetti, M. V. F. 1990, ApJ, 365, 164

Diaz, A. I., Terlevich, E., Pagel, B. E. J., Vilchez, J. M., \& Edmunds, M. G. 1987, MNRAS, 226, 19

Dodorico, S., \& Rosa, M. 1981, ApJ, 248, 1015

Filippenko, A. V. 1982, PASP, 94, 715

Foreman-Mackey, D., Hogg, D. W., Lang, D., \& Goodman, J. 2013, PASP, 125, 306
Genzel, R., Newman, S., Jones, T., et al. 2011, ApJ, 733, 101 González Delgado, R. M., \& Pérez, E. 2000, MNRAS, 317, 64 Guseva, N. G., Izotov, Y. I., \& Thuan, T. X. 2000, ApJ, 531, 776 Heckman, T. M., \& Borthakur, S. 2016, ApJ, 822, 9

Izotov, Y. I., Dyak, A. B., Chaffee, F. H., et al. 1996, ApJ, 458, 524

Lintott, C. J., Schawinski, K., Slosar, A., et al. 2008, MNRAS, 389, 1179 Lintott, C., Schawinski, K., Bamford, S., et al. 2011, MNRAS, 410, 166 Martin, C. L. 1999, ApJ, 513, 156

Planck Collaboration XVI. 2014, A\&A, 571, A16

Rodríguez del Pino, B., Arribas, S., Piqueras López, J., Villar-Martín, M., \& Colina, L. 2019, MNRAS, 486, 344

Roy, J.-R., Boulesteix, J., Joncas, G., \& Grundseth, B. 1991, ApJ, 367, 141

Roy, J.-R., Aube, M., McCall, M. L., \& Dufour, R. J. 1992, ApJ, 386, 498 Sargent, W. L. W., \& Filippenko, A. V. 1991, AJ, 102, 107

Terlevich, E., Díaz, A. I., Terlevich, R., et al. 1996, MNRAS, 279, 1219

Terlevich, R., Terlevich, E., Bosch, G., et al. 2014, MNRAS, 445, 1449

Villar Martín, M., Emonts, B., Humphrey, A., Cabrera Lavers, A., \& Binette, L. 2014, MNRAS, 440, 3202 